\title{
Common variants of $F T O$ and the risk of obesity and type 2 diabetes in Indians
}

\author{
Ganesh Chauhan $^{1,7}$, Rubina Tabassum ${ }^{1,7}$, Anubha Mahajan ${ }^{1}$, Om Prakash Dwivedi ${ }^{1}$, Yuvaraj Mahendran ${ }^{1}$, \\ Ismeet Kaur ${ }^{1}$, Shubhanchi Nigam ${ }^{1}$, Himanshu Dubey ${ }^{1}$, Binuja Varma ${ }^{2}$, Sri Venkata Madhu ${ }^{3}$, Sandeep \\ K Mathur ${ }^{4}$, Saurabh Ghosh ${ }^{5}$, Nikhil Tandon ${ }^{6}$ and Dwaipayan Bharadwaj ${ }^{1}$
}

Common variants of fat mass and obesity-associated gene (FTO, fat mass- and obesity-associated gene) have been shown to be associated with obesity and type 2 diabetes in population of European and non-European ethnicity. However, studies in Indian population have provided inconsistent results. Here, we examined association of eight FTO variants (rs1421085, rs8050136, rs9939609, rs9930506, rs1861867, rs9926180, rs2540769 and rs708277) with obesity and type 2 diabetes in 5364 North Indians (2474 type 2 diabetes patients and 2890 non-diabetic controls) in two stages. None of the variants including previously reported intron 1 variants (rs1421085, rs8050136, rs9939609 and rs9930506) showed body mass index (BMI)-dependent/ independent association with type 2 diabetes. However, rs1421085, rs8050136 and rs9939609 were associated with obesity status and measures of obesity (BMI, waist circumference and waist-to-hip ratio) in stage 2 and combined study population. Meta-analysis of the two study population results also revealed that rs 1421085 , rs 8050136 and rs9939609 were significantly associated with BMI both under the random- and fixed-effect models $(P$ (random/fixed) $=0.02 / 0.0001,0.004 / 0.0006$ and $0.01 / 0.01$, respectively). In conclusion, common variants of $F T O$ were associated with obesity, but not with type 2 diabetes in North Indian population.

Journal of Human Genetics (2011) 56, 720-726; doi:10.1038/jhg.2011.87; published online 4 August 2011

Keywords: diabetes; FTO; genetic variants; Indians; obesity

\section{INTRODUCTION}

Type 2 diabetes and obesity have reached epidemic proportions worldwide and the prevalence is increasing at rising rates, especially in developing countries. ${ }^{1,2}$ Both genetic and environmental factors with overlapping etiological mechanisms are believed to be involved in the development of obesity and type 2 diabetes. FTO (fat mass- and obesity-associated gene) is one of the best examples of diabetogenic gene mediating its effect through obesity. FTO was first identified as susceptibility gene for obesity ${ }^{3}$ and later body mass index (BMI)dependent association with type 2 diabetes was revealed in European population. ${ }^{4}$ Among the variants known to influence obesity, FTO variants had maximal effect in Europeans. ${ }^{5}$ Subsequently, a number of studies confirmed the association of common variants of FTO in population of European and non-European ethnicity. ${ }^{6-10}$ Independent studies in Asian population ${ }^{11-15}$ had yielded conflicting results, but a recent meta-analysis of common variants of FTO in East Asian population confirmed their association with obesity and type 2 diabetes. ${ }^{16}$

However, association of common variants of FTO in Indian population remains inconclusive. Two studies from Pune-Mysore ${ }^{17}$ and North Indian Sikhs ${ }^{18}$ demonstrated a BMI-independent association of rs9939609 with type 2 diabetes, but failed to replicate its association with obesity. A recent study on a South Indian population from Chennai ${ }^{19}$ demonstrated BMI-dependent association of rs8050136 with type 2 diabetes and also with obesity. These contradictions in association results for variants in FTO with obesity and type 2 diabetes demand their further validation in Indian population.

In this study, we evaluated association of previously associated common variants of FTO in intron 1 (rs9939609, rs1421085, rs8050136 and rs9930506) and additional variants (rs1861867intron 2, rs9926180 - intron 7, rs2540769-intron 8 and rs708277$3^{\prime}$-UTR) of FTO with type 2 diabetes and obesity in North Indians of Indo-European ethnicity.

\section{MATERIALS AND METHODS}

Study participants

Study involved participation of 5364 subjects from North India belonging to Indo-European ethnicity residing in and around Delhi, in two stages. In stage 1, association analysis was performed in 2115 subjects comprising of 1073 type 2

${ }^{1}$ Genomics and Molecular Medicine Unit, Institute of Genomics and Integrative Biology (CSIR), Delhi, India; ${ }^{2}$ The Centre of Genomics Applications, Okhla Industrial Area Phase III, New Delhi, India; ${ }^{3}$ Division of Endocrinology, University College of Medical Sciences, Delhi, India; ${ }^{4}$ Department of Endocrinology, SMS Medical College and Hospital, Rajasthan, India; ${ }^{5}$ Human Genetics Unit, Indian Statistical Institute, Kolkata, India and ${ }^{6}$ Department of Endocrinology, All India Institute of Medical Sciences, New Delhi, India

${ }^{7}$ These authors contributed equally to this work.

Correspondence: Professor N Tandon, Department of Endocrinology, All India Institute of Medical Sciences, Ansari Nagar, New Delhi 110029 , India.

E-mail: nikhil2811tandon@gmail.com

or Dr D Bharadwaj, Genomics and Molecular Medicine Unit, Institute of Genomics and Integrative Biology, CSIR, Mall Road, Delhi 110 007, India.

E-mail: db@igib.res.in

Received 20 February 2011; revised 18 June 2011; accepted 5 July 2011; published online 4 August 2011 
diabetes patients and 1042 controls, who were recruited before September 2008. All type 2 diabetes patients of stage 1 were recruited from the Clinic (Endocrinology Clinic, All India Institute of Medical Sciences), whereas control subjects were collected by organizing 'Diabetes Awareness Camps'. In stage 2, further replication was assessed in an independent sample set of 3249 individuals recruited after September 2008 that included 1401 type 2 diabetes patients (recruited from Clinic and 'Diabetes Awareness Camps') and 1848 controls (enrolled from 'Diabetes Awareness Camps'). Type 2 diabetes was diagnosed as per the World Health Organization criteria. ${ }^{20}$ Control subjects had age $\geqslant 40$ years, no family history of diabetes with glycosylated hemoglobin level $\leqslant 6.0 \%$ and fasting glucose level $\leqslant 6.11 \mathrm{mmoll}^{-1}$. Detailed inclusion and exclusion criteria for type 2 diabetes patients and control subjects have been provided earlier. ${ }^{21,22}$ Informed consent was obtained from all the participants and the study was approved by the Ethics committee of the participating institutions. The study was conducted in accordance with the principles of the Declaration of Helsinki.

\section{Clinical measurements}

All subjects were extensively characterized for anthropometric and quantitative metabolic traits. Anthropometric measurements including height, weight, waist circumference (WC), hip circumference, systolic and diastolic blood pressure were taken as per standardized protocols. BMI and waist-to-hip ratio (WHR) were calculated. Biochemical measurements including levels of glycosylated hemoglobin, fasting plasma glucose, 2-h post-load plasma glucose, fasting plasma insulin, C-reactive protein, C-peptide, total cholesterol (TC), high-density lipoprotein-cholesterol, low-density lipoprotein-cholesterol, triglyceride, blood urea, creatinine and uric acid were performed using standard laboratory assays as described earlier. ${ }^{21}$ Homeostasis model assessment for insulin resistance was calculated using the formula: (fasting plasma insulin $\left(\mathrm{mUl}^{-1}\right) \times$ fasting plasma glucose $\left.\left(\mathrm{mmoll}^{-1}\right)\right) / 22.5$ as reported previously. ${ }^{23}$ General characteristics of study population are presented in Table 1.

\section{SNP selection}

Four single-nucleotide polymorphisms (SNPs) from intron 1 (rs1421085, rs8050136, rs9939609, rs9930506) that were previously shown to be associated with obesity/type 2 diabetes were selected. In addition, four more SNPs (rs1861867-intron 2, rs9926180-intron 7, rs2540769-intron 8 and rs708277-3'-UTR) were selected from the database (NCBI dbSNP Build 129) to cover the gene at regular intervals. These SNPs were prioritized based on

Table 1 General characteristics of the study populations

\begin{tabular}{|c|c|c|c|c|c|c|}
\hline \multirow[b]{2}{*}{ Characteristics } & \multicolumn{2}{|c|}{ Stage 1} & \multicolumn{2}{|c|}{ Stage 2} & \multirow[b]{2}{*}{$\mathrm{P}$-value $\mathrm{a}^{\mathrm{a}}$} & \multirow[b]{2}{*}{$\mathrm{P}$-value ${ }^{\mathrm{b}}$} \\
\hline & Type 2 diabetes patients & Controls & Type 2 diabetes patients & Controls & & \\
\hline$N$ (men/women) & $1019(592 / 427)$ & $1006(606 / 400)$ & $1401(851 / 550)$ & $1848(1017 / 831)$ & - & - \\
\hline Age (years) & $53(45-62)$ & $50(44-60)$ & $55(48-62)$ & $52(45-62)$ & 0.03 & $1.9 \times 10^{-6}$ \\
\hline Age of diagnosis (years) & $45(39-52)$ & - & $47(40-55)$ & & $2.3 \times 10^{-4}$ & \\
\hline $\begin{array}{l}\text { Individuals on medication } \\
\text { for lipids (\%) }\end{array}$ & 4.00 & - & 7.35 & 0.87 & - & - \\
\hline \multicolumn{7}{|l|}{$B M I\left(\mathrm{~kg} \mathrm{~m}^{-2}\right)$} \\
\hline Women & $26.70(24.20-29.20)$ & 24.90 (21.10-28.60) & $27.34(24.46-31.00)$ & 26.30 (23.11-29.23) & 0.001 & $1.3 \times 10^{-5}$ \\
\hline Men & $23.80(22.00-26.00)$ & $23.20(20.20-25.70)$ & $25.40(22.94-28.36)$ & 24.69 (22.15-27.35) & $3.0 \times 10^{-15}$ & $3.7 \times 10^{-13}$ \\
\hline \multicolumn{7}{|l|}{$W C$} \\
\hline Women & $91.44(86.36-96.52)$ & $85.00(75.60-93.00)$ & 86.68 (40.00-98.00) & $88.00(81.00-95.50)$ & $3.8 \times 10^{-34}$ & $1.1 \times 10^{-5}$ \\
\hline Men & $86.36(86.36-91.44)$ & $88.50(80.64-95.00)$ & $90.00(36.00-98.00)$ & $93.00(86.00-100.00)$ & $8.4 \times 10^{-44}$ & $1.9 \times 10^{-16}$ \\
\hline \multicolumn{7}{|l|}{ WHR } \\
\hline Women & $1.00(0.97-1.03)$ & $0.86(0.82-0.92)$ & $0.95(0.89-0.98)$ & $0.87(0.82-0.91)$ & $5.9 \times 10^{-23}$ & 0.57 \\
\hline Men & $1.00(0.97-1.03)$ & $0.97(0.92-1.00)$ & $0.98(0.96-1.03)$ & $0.97(0.92-1.00)$ & 0.002 & 0.53 \\
\hline $\mathrm{FPG}\left(\mathrm{mmol} \mathrm{I}^{-1}\right)$ & $7.90(6.40-10.30)$ & $4.90(4.50-5.30)$ & 8.05 (6.39-10.60) & $4.82(4.41-5.20)$ & 0.28 & $2.8 \times 10^{-4}$ \\
\hline 2-h PPG (mmol I-1) & - & $5.60(5.80-6.30)$ & - & $5.76(4.95-6.55)$ & & 0.06 \\
\hline $\mathrm{HbAlc}(\%)$ & $7.80(6.50-9.40)$ & $5.20(4.90-5.60)$ & $8.19(6.90-9.70)$ & $5.70(5.40-5.90)$ & $2.6 \times 10^{-5}$ & $1.3 \times 10^{-114}$ \\
\hline $\mathrm{FPI}\left(\mathrm{pmol} \mathrm{I}^{-1}\right)$ & - & $32.20(17.50-57.20)$ & - & $43.68(28.20-63.36)$ & - & $4.1 \times 10^{-16}$ \\
\hline HOMA IR & - & $1.16(0.59-2.02)$ & - & $1.56(0.97-2.38)$ & - & $9.2 \times 10^{-15}$ \\
\hline C-peptide (nmol I-1) & $0.89(0.56-1.36)$ & $0.53(0.36-0.73)$ & $0.95(0.60-1.45)$ & $0.67(0.51-0.86)$ & $2.3 \times 10^{-9}$ & $3.6 \times 10^{-26}$ \\
\hline $\operatorname{hsCRP}\left(\left.m g\right|^{-1}\right)$ & $2.20(0.90-4.70)$ & $1.30(0.60-3.00)$ & $1.93(0.93-3.63)$ & $1.68(0.92-3.11)$ & $3.4 \times 10^{-5}$ & $8.8 \times 10^{-10}$ \\
\hline $\mathrm{TC}\left(\mathrm{mmoll} \mathrm{I}^{-1}\right)$ & $4.20(3.50-5.00)$ & $4.40(3.70-5.10)$ & $4.57(3.73-5.39)$ & $4.83(4.16-5.46)$ & 0.13 & $1.8 \times 10^{-25}$ \\
\hline HDL-C (mmol I-1) & $1.03(0.88-1.22)$ & $1.06(0.88-1.28)$ & $1.08(0.92-1.30)$ & $1.20(1.01-1.42)$ & $2.0 \times 10^{-7}$ & $1.2 \times 10^{-27}$ \\
\hline LDL-C (mmoll-1) & $2.57(1.99-3.36)$ & $2.79(2.33-3.41)$ & $2.70(2.04-3.40)$ & $2.97(2.46-3.49)$ & 0.009 & $2.5 \times 10^{-6}$ \\
\hline $\mathrm{TG}\left(\mathrm{mmol} \mathrm{I}^{-1}\right)$ & $1.60(1.10-2.20)$ & $1.30(1.00-1.80)$ & $1.39(0.91-2.11)$ & $1.16(0.81-1.62)$ & 0.84 & $1.2 \times 10^{-14}$ \\
\hline Urea $\left(\mathrm{mmol} \mathrm{I}^{-1}\right)$ & $9.28(7.13-11.83)$ & $8.5(6.78-10.31)$ & $9.5(7.57-12.50)$ & $8.57(6.96-10.42)$ & $7.7 \times 10^{-7}$ & 0.33 \\
\hline Uric acid $\left(\mu \mathrm{mol} \mathrm{I}^{-1}\right)$ & $291.45(231.97-356.88)$ & $291.45(238.51-341.42)$ & $289.37(236.14-350.93)$ & $303.35(248.63-365.80)$ & $7.6 \times 10^{-10}$ & $1.2 \times 10^{-4}$ \\
\hline Creatinine $\left(\mu \mathrm{mol} \mathrm{I}^{-1}\right)$ & $74.26(59.23-94.59)$ & $66.3(58.34-77.79)$ & $67.18(56.58-82.21)$ & $64.53(53.92-76.02)$ & $3.8 \times 10^{-5}$ & $1.0 \times 10^{-4}$ \\
\hline Systolic BP (mm Hg) & $130(130-140)$ & $120(112-133)$ & $130(122-140)$ & $128(120-140)$ & $7.6 \times 10^{-10}$ & $6.1 \times 10^{-5}$ \\
\hline Diastolic BP (mm Hg) & $80(78-90)$ & $80(70-88)$ & $82(80-90)$ & $80(80-90)$ & $3.9 \times 10^{-5}$ & $5.7 \times 10^{-17}$ \\
\hline
\end{tabular}

Abbreviations: BMI, body mass index; BP, blood pressure; FPG, fasting plasma glucose; FPI, fasting plasma insulin; HbAlc, glycosylated hemoglobin Alc; HDL-C, high-density lipoprotein cholesterol; HOMA-IR, homeostasis model assessment for insulin resistance; hsCRP, high-sensitivity C-reactive protein; LDL-C, low-density lipoprotein cholesterol; N, number of individuals; 2-h PPG, 2-h post-load plasma glucose; TC, total cholesterol; TG, triglycerides; WC, waist circumference; WHR, waist-to-hip ratio.

PPG, 2-h post-load plasma glucose; TC, total cholester
Data are presented as median (interquartile range).

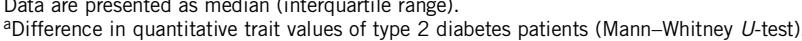

bifference in quantitative trait values of controls (Mann-Whitney U-test). 
their minor allele frequency (MAF) ( $>0.05$ ), polymorphic state in at least two population and localization in functionally important region.

\section{Genotyping}

Genotyping in stage 1 was performed by Golden Gate assay using Illumina platform (http://www.illumina.com/documents/products/workflows/workflow_ goldengate_assay.pdf). Stringent quality control criteria were met to qualify an SNP for further analysis, including genotype confidence score $>0.25$, call frequency $>0.9$, GenTrans score $>0.6$, cluster separation score $>0.4$, MAF $>0.05$ and Hardy-Weinberg equilibrium $P>0.01$ in controls. ${ }^{24} \mathrm{~A}$ total of 147 samples $(7.2 \%)$ were also genotyped in duplicate and an error rate of $<0.01$ was estimated. The variant rs708277 did not qualify the quality control (GenTrans score $<0.6$ ) and was excluded from further analysis.

In stage 2, genotyping of rs9926180 (associated in stage 1) and rs9939609 (established obesity/type 2 diabetes variant in stage 2) was performed in 1047 cases and 1038 controls using iPLEX (Sequenom, San Diego, CA, USA). Approximately $5 \%(N=110)$ of the samples of stage 2 were genotyped in duplicates and a concordance of $99.99 \%$ was observed. In addition, 810 controls were genotyped for all the seven SNPs of stage 1 and 354 cases for rs9939609 and rs9926180, to increase power of the study. These samples were genotyped by single base extension using SNaPshot ddNTP Primer Extension Kit (Applied Biosystems, Foster City, CA, USA) and electrophoresis of extended probes on an ABI Prism 3730 Genetic Analyzer (Applied Biosystems). The genotype calls were determined using GeneMapper. Approximately $6 \%$ of the samples were genotyped in duplicates and a concordance of $99.99 \%$ was observed. SNaPshot assay for two SNPs rs1861867 and rs2540769 failed in this phase.

\section{Statistical analysis}

Statistical analyses were performed using PLINK v. 1.07 (http://pngu.mgh. harvard.edu/purcell/plink/25) and SPSS v. 17.0 (SPSS, Chicago, IL, USA), unless specified otherwise. Statistical power of the study was calculated under logadditive model, assuming 10\% population risk using Quanto (http://hydra. usc.edu/gxe/) (Supplementary Tables 1 and 2, Supplementary Figure 1). Hardy-Weinberg equilibrium for genotypes was checked by $\chi^{2}$ analysis. Linkage disequilibrium between SNPs was estimated using Haploview v. $4.1 .^{26}$ Single marker- and haplotype-based association with obesity and type 2 diabetes was assessed by logistic regression analysis assuming log-additive model. A $P$-value of $<0.01(\alpha=0.05 / 5)$ was considered significant after Bonferroni correction for the number of independent SNPs investigated. All quantitative trait values were inverse normally transformed to achieve normal distribution and then analyzed using linear regression. Means and standard deviations are presented in inverse normal units of the parameters in the tables. For quantitative traits association, a $P$-value of $<0.00053(\alpha=0.05 /(5 \times 19))$ was considered significant (association for five independent loci with 19 traits). Association with obesity and quantitative traits was performed only in control subjects.

Meta-analysis of stage 1 and 2 results were performed by combining summary data of two study population both under fixed and random models. Similarly, the summary data of previous studies on Indians for association with type 2 diabetes ${ }^{17-19}$ and this study were combined for meta-analysis. Association of variants with type 2 diabetes, obesity and quantitative traits were also performed by combining data for two study population and adjusting for study population in addition to other covariates. Non-parametric MannWhitney $U$-test was performed to compare the median values of clinical traits of two study population. Allele frequencies of cases and controls of two study population were compared by equality of proportions $Z$-test (Supplementary Table 3).

All analyses performed were adjusted for age, sex and BMI as appropriate. The odds ratios (ORs) and 95\% confidence interval (CI) were calculated with respect to minor alleles and uncorrected $P$-values are presented in the manuscript.

\section{RESULTS}

\section{Association with type 2 diabetes}

In stage 1, rs9926180 (intron 7) was associated with type 2 diabetes that remained significant after adjusting for sex, age and BMI $\left(\mathrm{OR}_{\mathrm{adj}}=1.24,95 \% \mathrm{CI}=1.07-1.43, P_{\mathrm{adj}}=0.005\right)$ and even after correcting for multiple tests (Table 2). However, association of rs9926180

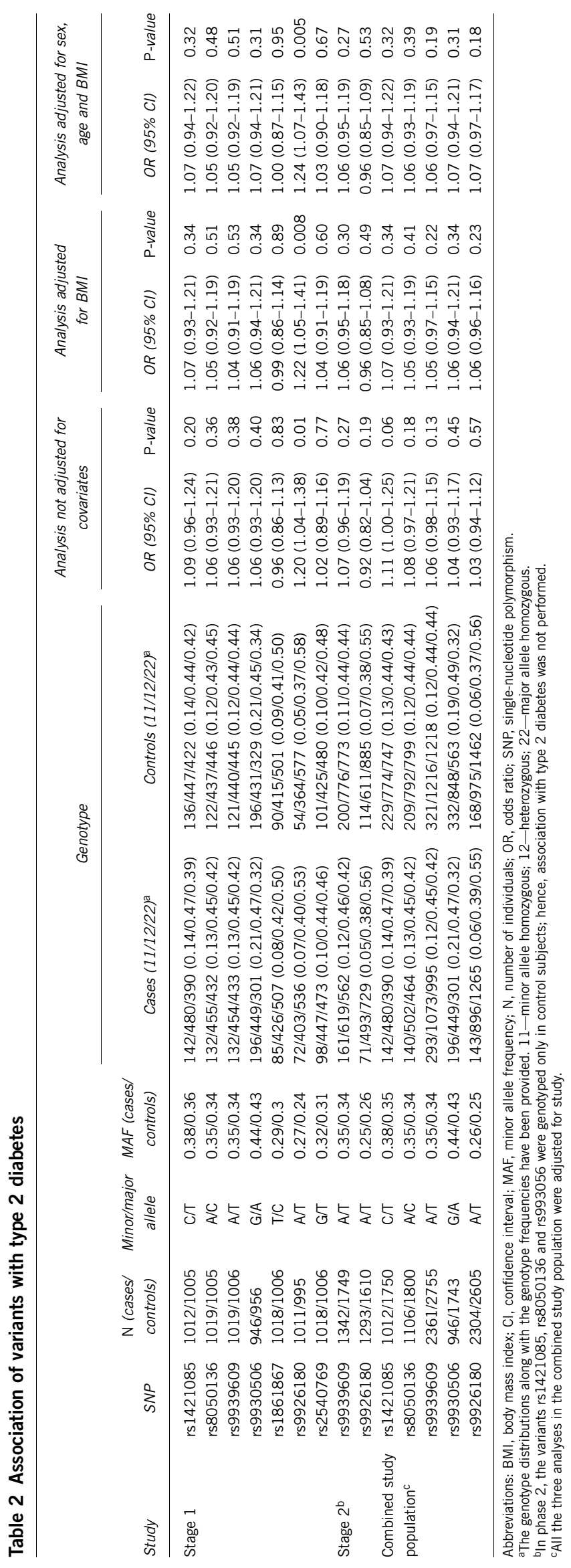


could not be replicated in another independent study population in stage 2. Meta-analysis and combined analysis of two stages also did not show association of rs9926180 with type 2 diabetes (Table 4). None of the previously reported SNPs showed either BMI-dependent or -independent association with type 2 diabetes in our population. Interestingly, the most widely studied variant rs9939609 also failed to show any association with type 2 diabetes in our study population. Similar results were obtained when association analysis were performed by adjusting for sex, age and BMI as covariates and only BMI as a covariate (Table 2).

\section{Association with obesity and its measures}

For association analysis with obesity, control subjects were categorized as normal weight (BMI $<23 \mathrm{~kg} \mathrm{~m}^{-2}$ ), overweight (BMI 23$25 \mathrm{~kg} \mathrm{~m}^{-2}$ ) and obese (BMI $\geqslant 25 \mathrm{~kg} \mathrm{~m}^{-2}$ ) based on BMI cutoffs for Asians defined by World Health Organization expert committee ${ }^{27}$ (Supplementary Table 4). None of the variants showed association with BMI-defined obesity in stage 1 of the study (Table 3). However, in stage $2, \mathrm{rs} 1421085(\mathrm{OR}=1.57,95 \% \mathrm{CI}=1.21-2.04, P=0.0007)$ and rs8050136 $(\mathrm{OR}=1.37,95 \% \mathrm{CI}=1.06-1.77, P=0.02)$ showed association with obesity when we compared obese/overweight with normal weight subjects. In the same stage, the association was also observed when we compared obese with normal weight subjects (rs1421085: $\mathrm{OR}=1.65,95 \% \mathrm{CI}=1.26-2.17, P=0.0003 ; \mathrm{rs} 8050136: \mathrm{OR}=1.45,95 \%$ $\mathrm{CI}=1.11-1.91, P=0.007)$ with increased risk. The associations were also observed after combined analysis and meta-analysis (Table 4). As WC has been shown to be a better predictor of obesity, ${ }^{28}$ we also tested association by defining obesity using WC $(>90 \mathrm{~cm}$ in men and $>80 \mathrm{~cm}$ in women $)^{28}$ and WHR $(>0.95$ in men and $>0.80$ in women $)^{29}$ as measures of central obesity (Supplementary Table 5). The same variant rs1421085 was associated with WC-defined obesity in stage $2(\mathrm{OR}=1.43,95 \% \mathrm{CI}=1.11-1.85, P=0.006)$ and combined study population $(\mathrm{OR}=1.23,95 \% \mathrm{CI}=1.06-1.43, P=0.006)$. In WHRdefined obesity analysis, rs 1421085 and rs9930506 showed nominal association only in stage 2 of the study. Association analysis with quantitative measures of obesity (BMI, WC and WHR) in stage 1 showed only nominal association of $\operatorname{rs} 1421085(P=0.04)$, rs8050136 $(P=0.03)$ and rs9939609 $(P=0.03)$ with WC (Supplementary Table 6). However in stage 2 (Supplementary Table 7), association with BMI and WC was observed for rs1421085 (BMI: $P=0.0002$; WC: $P=0.001$ ), rs8050136 (BMI: $P=0.002$; WC: $P=0.05$ ) and rs9930506 (BMI: $P=0.04$; WC: $P=0.02)$. The variant rs9939609 also showed nominal association with WC $(P=0.03)$. In the combined study population (Supplementary Table 8), all the above three variants were associated with BMI and WC in addition to nominal association of rs9939609 with WHR $(P=0.03)$.

\section{Association with quantitative trait}

We also examined association of FTO variants with other quantitative traits related to obesity and type 2 diabetes to see if they affect risk of obesity/type 2 diabetes by influencing them. In stage 1, rs9926180 was associated with high-density lipoprotein-cholesterol $(P=0.002)$, and in stage 2, rs9930506 was associated with triglycerides $(P=0.002)$. Nominal associations of FTO variants with other traits were also observed (Supplementary Table 6).

\section{Haplotype-based association}

Intron 1 variants-rs1421085, rs8050136 and rs9939609 formed a haplotype block encompassing a region of $19 \mathrm{~kb}$ (Supplementary Figure 2). Association analysis of this haplotype block with type 2

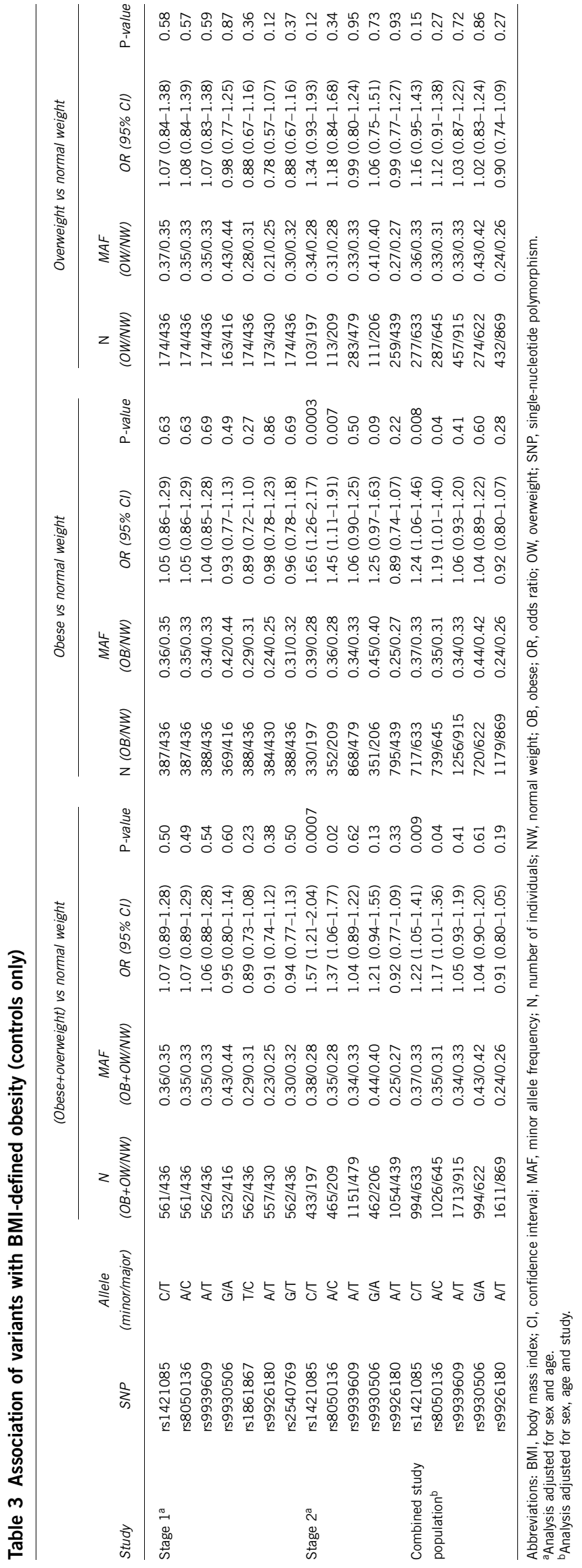


Table 4 Meta-analysis for the two study populations

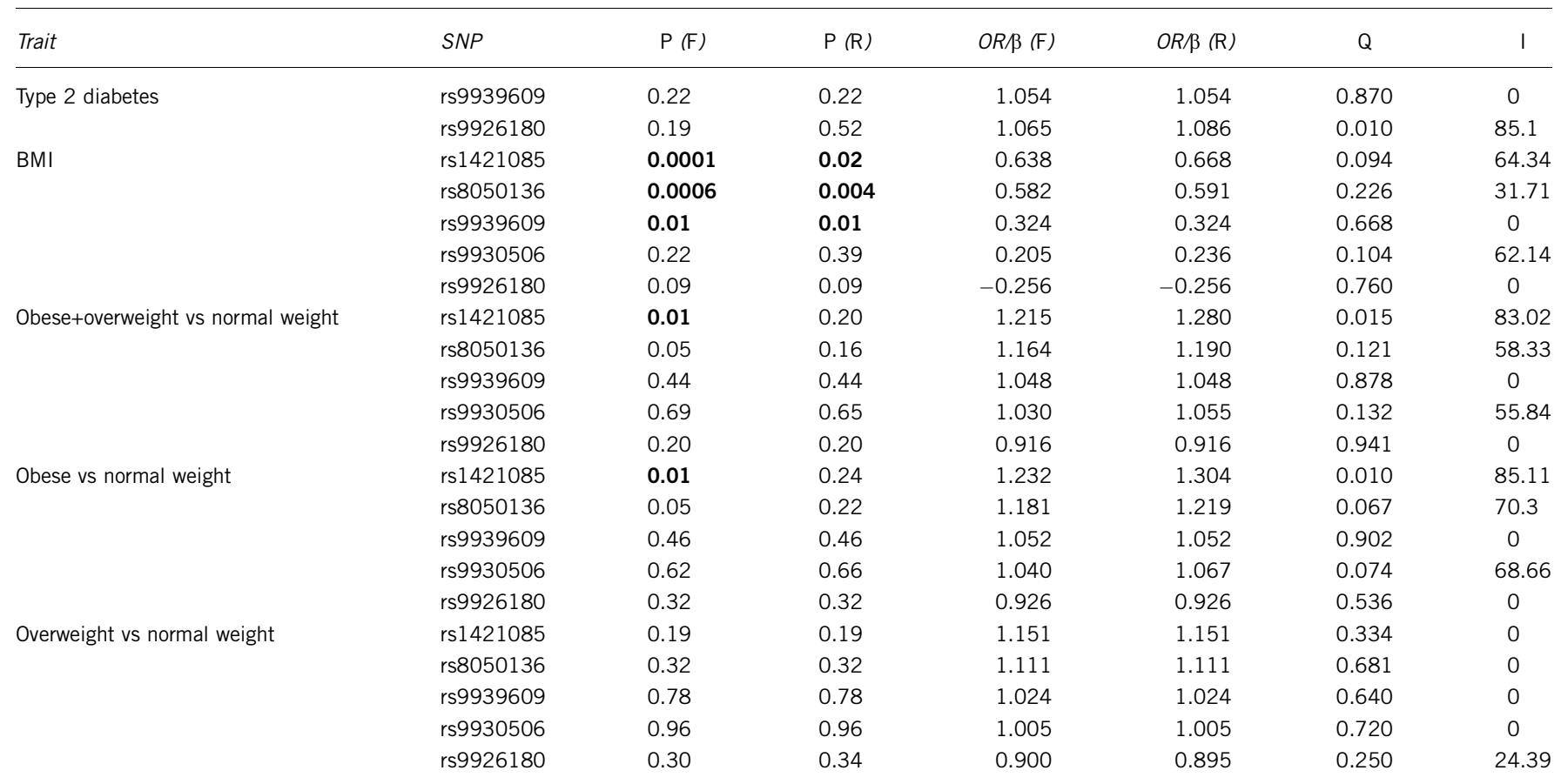

Abbreviations: P (F), fixed-effect meta-analysis $P$-value; $\mathrm{P}(\mathrm{R})$, random-effect meta-analysis $P$-value; OR (F), fixed-effect OR estimate; OR (R), random-effect OR estimate; $Q, P$-value for Cochrane's $Q$ statistic; I, $\wedge^{\wedge} 2$ heterogeneity index (0-100).

$P$-value in bold are significant.

diabetes, obesity and BMI failed to show any association (Supplementary Table 9).

\section{Meta-analysis with other studies}

Meta-analysis of the four studies on Indians (including this study) for association with type 2 diabetes (Figure 1) showed association under the random-effect $\left(\mathrm{OR}_{\text {random }}=1.16, P\right.$-value random $\left.=0.02\right)$ and fixedeffect models $\left(\mathrm{OR}_{\text {random }}=1.21, P\right.$-value random $\left.=0.001\right)$.

\section{DISCUSSION}

Here we explored association of FTO variants with type 2 diabetes, obesity and related quantitative traits in North Indians. We did not find FTO variants to influence the risk of type 2 diabetes in our study population. Our preliminary study suggested a BMI-independent association of a new variant rs9926180 in intron 7 with type 2 diabetes, but that could not be replicated in an independent study population. However, variants of intron 1 (rs1421085, rs8050136 and rs9939609) were associated with obesity status and/or measures of obesity (BMI, WC and WHR). This is in accordance with previous reports of these variants for association with obesity.

It is pertinent to mention that consistent with results of the studies in other population, our previous study demonstrated association of top eight genome-wide association studies confirmed loci with type 2 diabetes in North Indian population..$^{30}$ However, in this study, we failed to observe association for FTO in the same study population. Phenotypic differences between Indians and Europeans might be one of the possible reasons for observed difference in association in two populations. Indians have higher body fat and lower muscle mass for a given BMI compared with Europeans, have different fat distribution (high truncal, subcutaneous and intra-abdominal fat) and are centrally obese. ${ }^{31}$ Studies in different ethnic groups of Indian population have provided contradictory reports for association of FTO variants with type 2 diabetes and obesity. A study that recruited subjects from Western and Southern India (Pune-Mysore study), ${ }^{17}$ representing Indo-European and Dravidian population, found rs9939609 to be associated with type 2 diabetes independent of BMI (MAF in controls 0.30). Similar results were obtained in another study of IndoEuropean population of North Indian Sikhs ${ }^{18}$ (MAF in controls 0.31). However, these studies failed to replicate association of rs9939609 with obesity in the control subjects. A recent study in Dravidian population from India ${ }^{19}$ demonstrated FTO variant association with type 2 diabetes independent of BMI and also with obesity in the control subjects (MAF in controls 0.11). However, they did not study rs9939609; instead, they studied rs8050136 (a proxy SNP for rs9939609). We investigated both these variants (rs9939609 and rs8050136) in this study and observed them to be in strong linkage disequilibrium $\left(r^{2}>0.99, D^{\prime}>0.99\right)$ with each other (MAF in controls 0.34 ). By far this is the largest study on any Indian population, investigating the role of FTO variants with obesity and type 2 diabetes. Despite being sufficiently powered, our study did not detect association of common variants of FTO with type 2 diabetes (dependent or independent of BMI). We also meta-analyzed the data of all the four studies on the Indian population (including this study) and found variants of FTO to be associated with type 2 diabetes, but with a lower effect size (Figure 1). This may be indicative of a lower effect size of FTO variants in this study population.

The contradictory findings of the studies in diverse ethnic groups from different geographical regions of India might indicate ethnicspecific effect of FTO variants in Indians. However, study design, study population and sample size can significantly affect the outcome of association studies. There was considerable heterogeneity in subject recruitment criteria, statistical analyses, sample sizes and most importantly sample collection areas among these studies on Indian population. The study in North Indian Sikhs involved participation of 
a

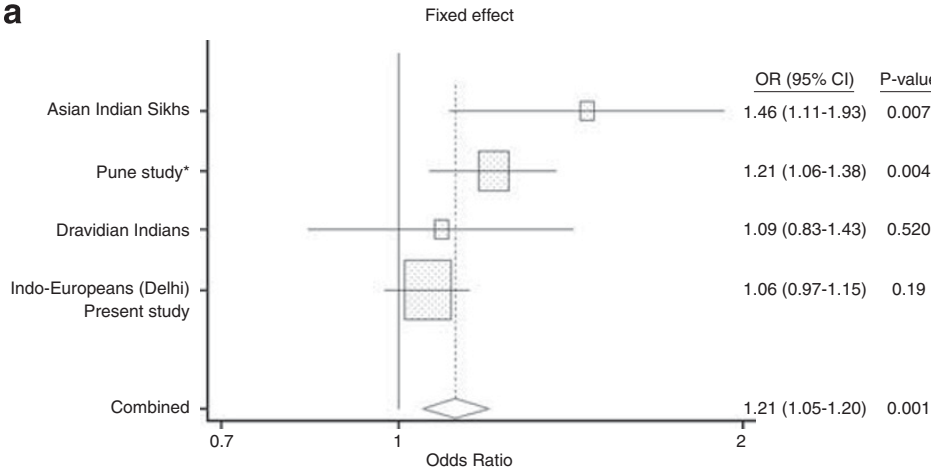

b

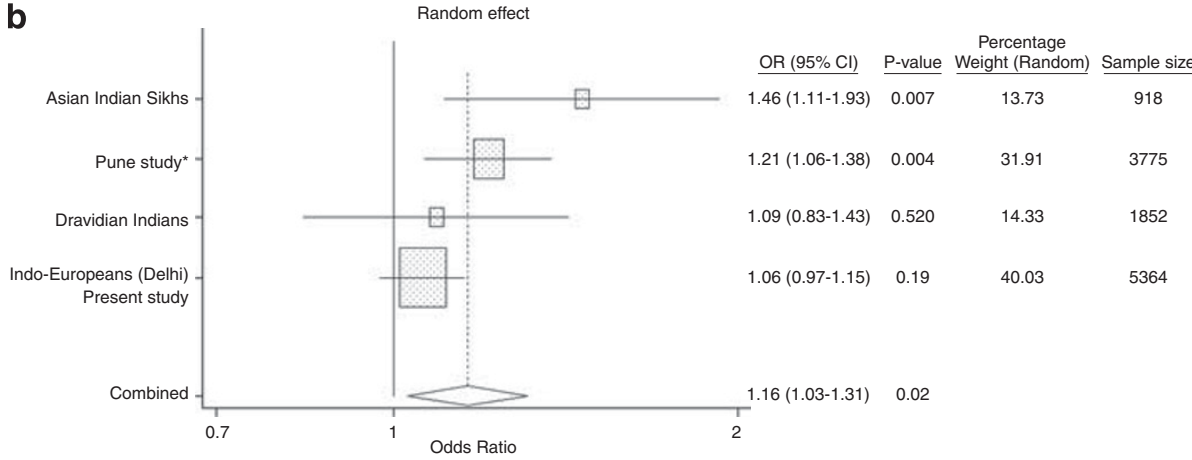

Figure 1 Forest plot of meta-analysis (fixed and random effects) for association of rs9939609 with type 2 diabetes in Indians. (a) Fixed effect and (b) random effect. *Pune study ${ }^{17}$ involved participation of both Indo-European (2814) and Dravidian Indians (961). In all studies, analysis was adjusted for sex, age and body mass index (BMI), except for Indo-Europeans (Pune), where analysis was adjusted only for BMI. For Dravidian Indians, ${ }^{19}$ rs8050136 was considered as proxy single-nucleotide polymorphism (SNP) for rs9939609 for this analysis as this SNP was not studied in Dravidian Indians. Test for heterogeneity: $Q=6.571$ on 3 d.f. $(P=0.09)$. Moment-based estimate between studies variance $=0.008$.

only 920 subjects. While the Pune study involved participation of 3775 subjects (inclusive of individuals of two different ethnicities: IndoEuropean and Dravidians) and for reasons unknown analysis was not adjusted for sex and age. Diet and physical activity are shown to have considerable effect in modulating the susceptibility to obesity by FTO variants. $^{32,33}$ There is tremendous diversity in diet and physical activity not only among Europeans and Indians, but also within different ethnic groups of Indian population. Hence, differences in genetic susceptibility of obesity and type 2 diabetes observed in different population might be due to modulation by populationspecific environmental factors and lifestyle. However, absence of data on diet and physical activity in this study population does not allow affirmative conclusion in this regard.

Although we observed differences in various anthropometric and biochemical measures of subjects in stage 1 and stage 2, there is no genetic heterogeneity between the subjects of the two stages (unpublished data). We performed a multidimensional scaling (MDS) analysis based on 608 unlinked markers genotyped in the initial study population of the present study that clearly demonstrates that our study population belongs to one cluster (Supplementary Figure 3 ). In stage 1 , all type 2 diabetes patients were recruited from the Clinic, and thus having longer duration of diabetes, under hypoglycemic and lipid-lowering medication that can influence quantitative measurements. Although stage 2 comprised of a combination of patients from the Clinic and 'Diabetes Awareness Camps' that included a proportion of patients who were newly detected with type 2 diabetes and without any kind of medication, this might result in the observed differences in glycemic measures, lipid profile and creatinine levels of patients in two stages. 'Diabetes Awareness Camps' in stage 1 were held in areas of low socio-economic localities, whereas stage 2 predominantly comprised of subjects recruited from camps organized in more affluent localities. We believe that difference in socio-economic localities of the subjects could explain the differences in the anthropometric and biochemical measures.

In conclusion, common variants of FTO were associated with obesity, but not with type 2 diabetes in our study population. However, it is also possible that the studied variants had small effects on type 2 diabetes, and that variants of FTO other than the ones studied are involved in conferring genetic susceptibility of type 2 diabetes and modulations of their effect through environment (diet and physical activity). Hence, further large-scale studies on Indians of different ethnicities with data for diet and physical activity and dense SNP coverage (end-to-end tag SNPs) spanning the entire gene is required to elucidate the role of FTO on the predisposition to type 2 diabetes in Indians.

\section{CONFLICT OF INTEREST}

The authors declare no conflict of interest.

\section{ACKNOWLEDGEMENTS}

We thank all the participants who volunteered for the study. We also thank Mr AK Sharma for his help in sample collection. This study was supported by 'Diabetes mellitus-New drug discovery R\&D, molecular mechanisms and genetic and epidemiological factors' (NWP0032-11) funded by Council of Scientific and Industrial Research (CSIR), Government of India. We thank Dr Mitali Mukerji (IGIB) and Dr Anurag Agrawal (IGIB) for critical evaluation of the manuscript. 
1 Wild, S., Roglic, G., Green, A., Sicree, R. \& King, H. Global prevalence of diabetes: estimates for the year 2000 and projections for 2030. Diabet. Care 27, 1047-1053 (2004).

2 Misra, A. \& Khurana, L. Obesity and the metabolic syndrome in developing countries. J. Clin. Endocrinol. Metab. 93, S9-S30 (2008).

3 Frayling, T. M., Timpson, N. J., Weedon, M. N., Zeggini, E., Freathy, R. M., Lindgren, C. M. et al. A common variant in the FTO gene is associated with body mass index and predisposes to childhood and adult obesity. Science 316, 889-894 (2007).

4 Scott, L. J., Mohlke, K. L., Bonnycastle, L. L., Willer, C. J., Li, Y., Duren, W. L. et al. Genome-wide association study of type 2 diabetes in Finns detects multiple susceptibility variants. Science $\mathbf{3 1 6}, 1341-1345$ (2007).

5 Loos, R. J. \& Bouchard, C. FTO: the first gene contributing to common forms of human obesity. Obes. Rev. 9, 246-250 (2008).

6 Scuteri, A., Sanna, S., Chen, W. M., Uda, M., Albai, G., Strait, J. et al. Genome-wide association scan shows genetic variants in the FTO gene are associated with obesityrelated traits. PLoS Genet. 3, e115 (2007).

7 Legry, V., Cottel, D., Ferrières, J., Arveiler, D., Andrieux, N., Bingham, A. et al. Effect of an FTO polymorphism on fat mass, obesity, and type 2 diabetes mellitus in the French MONICA Study. Metabolism 58, 971-975 (2009).

8 Adeyemo, A., Chen, G., Zhou, J., Shriner, D., Doumatey, A., Huang, H. et al. FTO genetic variation and association with obesity in West Africans and African Americans. Diabetes 59, 1549-1554 (2010).

9 Tönjes, A., Zeggini, E., Kovacs, P., Böttcher, Y., Schleinitz, D., Dietrich, K. et al. Association of FTO variants with BMI and fat mass in the self-contained population of Sorbs in Germany. Eur. J. Hum. Genet. 18, 104-110 (2010).

10 Zhang, G., Karns, R., Narancic, N. S., Sun, G., Cheng, H., Missoni, S. et al. Common SNPs in FTO gene are associated with obesity related anthropometric traits in an island population from the eastern Adriatic coast of Croatia. PLoS One 28, e10375 (2010).

11 Hotta, K., Nakata, Y., Matsuo, T., Kamohara, S., Kotani, K., Komatsu, R. et al. Variations in the FTO gene are associated with severe obesity in the Japanese. J. Hum. Genet. 53, 546-553 (2008).

12 Horikoshi, M., Hara, K., Ito, C., Shojima, N., Nagai, R., Ueki, K. et al. Variations in the HHEX gene are associated with increased risk of type 2 diabetes in the Japanese population. Diabetologia 50, 2461-2466 (2007).

13 Omori, S., Tanaka, Y., Takahashi, A., Hirose, H., Kashiwagi, A., Kaku, K. et al. Association of CDKAL1, IGF2BP2, CDKN2A/B, HHEX, SLC3OA8, and KCNJ11 with susceptibility to type 2 diabetes in a Japanese population. Diabetes 57, 791-795 (2008).

14 Chang, Y. C., Liu, P. H., Lee, W. J., Chang, T. J., Jiang, Y. D., Li, H. Y. et al. Common variation in the fat mass and obesity-associated (FTO) gene confers risk of obesity and modulates BMI in the Chinese population. Diabetes 57, 2245-2252 (2008).

15 Li, H., Wu, Y., Loos, R. J., Hu, F. B., Liu, Y., Wang, J. et al. Variants in the fat mass- and obesity-associated (FTO) gene are not associated with obesity in a Chinese Han population. Diabetes 57, 264-268 (2008).

16 Liu, Y., Liu, Z., Song, Y., Zhou, D., Zhang, D., Zhao, T. et al. Meta-analysis added power to identify variants in FTO associated with type 2 diabetes and obesity in the Asian population. Obesity 18, 1619-1624 (2010).
17 Yajnik, C. S., Janipalli, C. S., Bhaskar, S., Kulkarni, S. R., Freathy, R. M., Prakash, S. et al. FTO gene variants are strongly associated with type 2 diabetes in South Asian Indians. Diabetologia 52, 247-252 (2009).

18 Sanghera, D. K., Ortega, L., Han, S., Singh, J., Ralhan, S. K., Wander, G. S. et al. Impact of nine common type 2 diabetes risk polymorphisms in Asian Indian Sikhs: PPARG2 (Pro12Ala), IGF2BP2, TCF7L2 and FTO variants confer a significant risk. BMC Med. Genet. 9, 59 (2008).

19 Ramya, K., Radha, V., Ghosh, S., Majumder, P. P. \& Mohan, V. Genetic variations in the FTO gene are associated with type 2 diabetes and obesity in south Indians (CURES-79). Diabetes Technol. Ther. 13, 33-42 (2011).

20 Expert Committee on the Diagnosis and Classification of Diabetes Mellitus. Report of the expert committee on the diagnosis and classification of diabetes mellitus. Diabetes Care 26, S5-S20 (2003).

21 Tabassum, R., Chavali, S., Dwivedi, O. P., Tandon, N. \& Bharadwaj, D. Genetic variants of FOXA2: risk of type 2 diabetes and effect on metabolic traits in North Indians. J. Hum. Genet. 53, 957-965 (2008).

22 Mahajan, A., Tabassum, R., Chavali, S., Dwivedi, O. P., Bharadwaj, M., Tandon, N. et al. High-sensitivity C-reactive protein levels and type 2 diabetes in urban North Indians. J. Clin. Endocrinol. Metab. 94, 2123-2127 (2009).

23 Matthews, R. D., Hosker, J. P., Rudenski, A. S., Naylor, B. A., Treacher, D. F. \& Turner, R. C. Homeostasis model assessment: insulin resistance and $\beta$ cell function from fasting plasma glucose and insulin concentrations in man. Diabetologia 28, 412-419 (1985).

24 Mahajan, A., Tabassum, R., Chavali, S., Dwivedi, O. P., Chauhan, G., Tandon, N. et al. Obesity-dependent association of TNF-LTA locus with type 2 diabetes in North Indians. J. Mol. Med. 88, 515-522 (2010).

25 Purcell, S., Neale, B., Todd-Brown, K., Thomas, L., Ferreira, M. A., Bender, D. et al. PLINK: a tool set for whole-genome association and population-based linkage analyses. Am. J. Hum. Genet. 81, 559-575 (2007).

26 Barrett, J. C., Fry, B., Maller, J. \& Daly, M. J. Haploview: analysis and visualization of LD and haplotype maps. Bioinformatics 21, 263-265 (2005).

27 WHO Expert Consultation. Appropriate body-mass index for Asian populations and its implications for policy and intervention strategies. Lancet 363, 157-163 (2004).

28 Misra, A., Wasir, J. S. \& Pandey, R. M. An evaluation of candidate definitions of the metabolic syndrome in adult Asian Indians. Diabet. Care 28, 398-403 (2005).

29 Willett, W. C., Dietz, W. H. \& Colditz, G. A. Guidelines for healthy weight. N. Engl. J. Med. 341, 427-434 (1999).

30 Chauhan, G., Spurgeon, C. J., Tabassum, R., Bhaskar, S., Kulkarni, S. R., Mahajan, A. et al. Impact of common variants of PPARG, KCNJ11, TCF7L2, SLC30A8, HHEX, CDKN2A, IGF2BP2 and CDKAL1 on the risk of type 2 diabetes in 5164 Indians. Diabetes 59, 2068-2074 (2010).

31 Misra, A. \& Khurana, L. Obesity-related non-communicable diseases: South Asians vs White Caucasians. Int. J. Obes. 35, 167-187 (2011).

32 Sonestedt, E., Roos, C., Gullberg, B., Ericson, U., Wirfält, E. \& Orho-Melander, M. Fat and carbohydrate intake modify the association between genetic variation in the FTO genotype and obesity. Am. J. Clin. Nutr. 90, 1418-1425 (2009).

33 Vimaleswaran, K. S., Li, S., Zhao, J. H., Luan, J., Bingham, S. A., Khaw, K. T. et al. Physical activity attenuates the body mass index-increasing influence of genetic variation in the FTO gene. Am. J. Clin. Nutr. 90, 425-428 (2009).

Supplementary Information accompanies the paper on Journal of Human Genetics website (http://www.nature.com/jhg) 